\title{
Erratum
}

\section{Erratum to "Common Fixed Point Theorems in Modified Intuitionistic Fuzzy Metric Spaces"}

\author{
Saurabh Manro, ${ }^{1}$ Sanjay Kumar, ${ }^{2}$ S. S. Bhatia, ${ }^{1}$ and Kenan Tas $^{3}$ \\ ${ }^{1}$ School of Mathematics and Computer Applications, Thapar University, Patiala, 147001 Punjab, India \\ ${ }^{2}$ Deenbandhu Chhotu Ram University of Science and Technology, Murthal, Sonepat, 131027 Haryana, India \\ ${ }^{3}$ Department of Mathematics and Computer Science, Cankaya University, 06810 Ankara, Turkey \\ Correspondence should be addressed to Saurabh Manro; sauravmanro@hotmail.com
}

Received 22 November 2013; Accepted 5 December 2013; Published 30 January 2014

Copyright (C) 2014 Saurabh Manro et al. This is an open access article distributed under the Creative Commons Attribution License, which permits unrestricted use, distribution, and reproduction in any medium, provided the original work is properly cited.

On critical examination of the results given in our paper entitled "Common fixed point theorems in modified intuitionistic fuzzy metric spaces," we notice one crucial error. We need to carry out the following correction.

Example 22 given in the paper is wrong as follows.

None of the maps in $A, B, S$, and $T$ is continuous. Therefore, all conditions of Theorem 20 are not satisfied.

Hence, Example 22 in the paper is replaced by the below example.

Example 22. Let $X=[0, \infty)$ and for each $t>0$, define

$$
\zeta_{M, N}(x, y, t)=\left(\frac{t}{t+|x-y|}, \frac{|x-y|}{t+|x-y|}\right) \quad \forall x, y \in X
$$

Then $\left(X, \zeta_{M, N}, \mathscr{T}\right)$ is a complete modified intuitionistic fuzzy metric space. Let $A, B, S$, and $T$ be self-maps on $X$ defined as: $A x=B x=3 x / 4$ and $S x=T x=2 x$ for all $x \in X$. Clearly,

(i) the pairs $\{A, S\}$ and $\{B, T\}$ are continuous self-mappings on $X$;

(ii) $A(X) \subseteq T(X), B(X) \subseteq S(X)$;

(iii) $\{A, S\}$ and $\{B, T\}$ are $R$-weakly commuting pairs as both pairs commute at coincidence points;

(iv) $\{A, S\}$ and $\{B, T\}$ satisfy inequality (5) for all $x, y \in X$, where $k \in(0,1)$ and $\alpha=1$.

Hence, all conditions of Theorem 20 are satisfied and $x=$ 0 is a unique common fixed point of $A, B, S$, and $T$. 


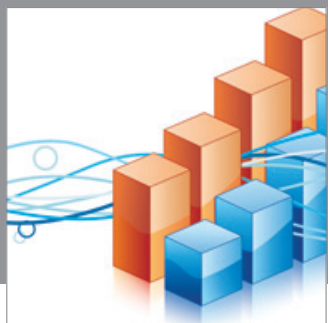

Advances in

Operations Research

mansans

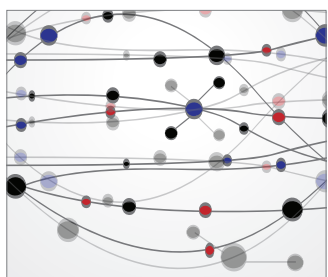

The Scientific World Journal
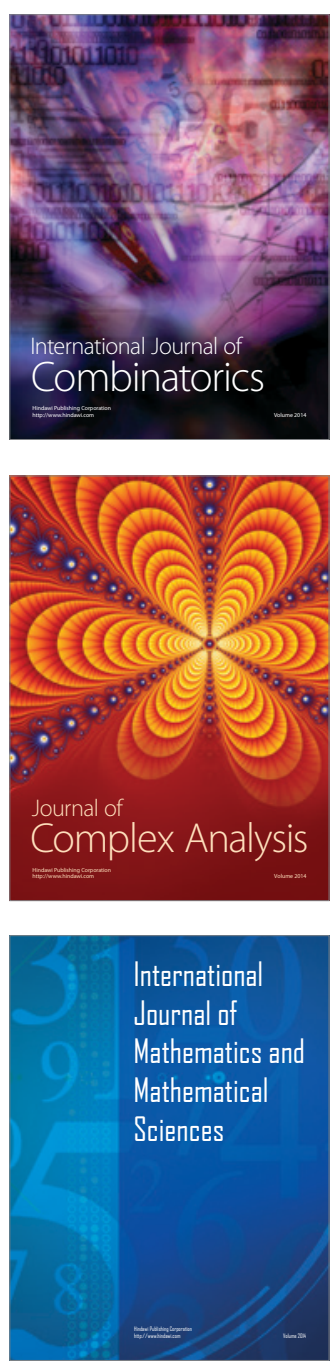
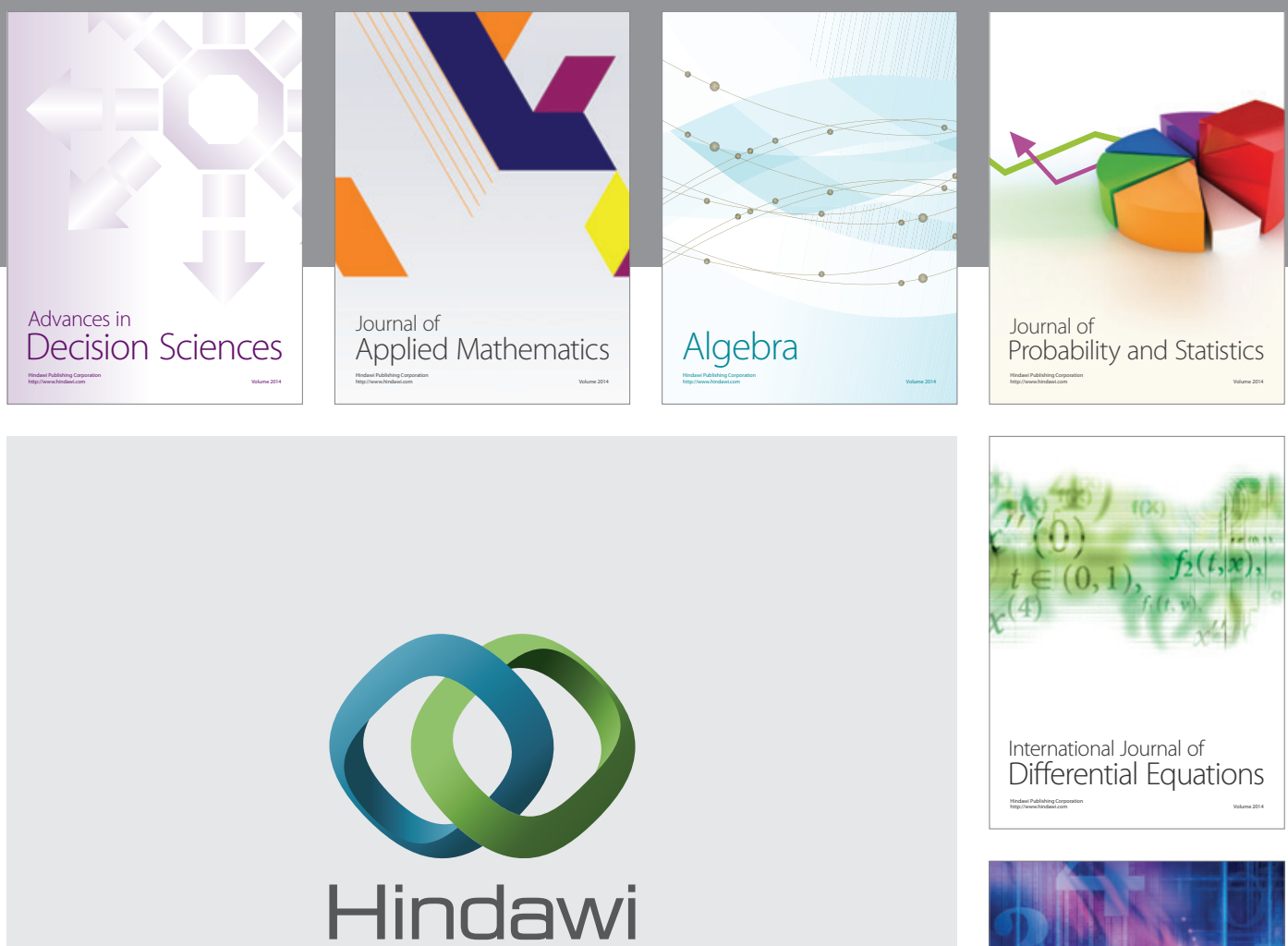

Submit your manuscripts at http://www.hindawi.com
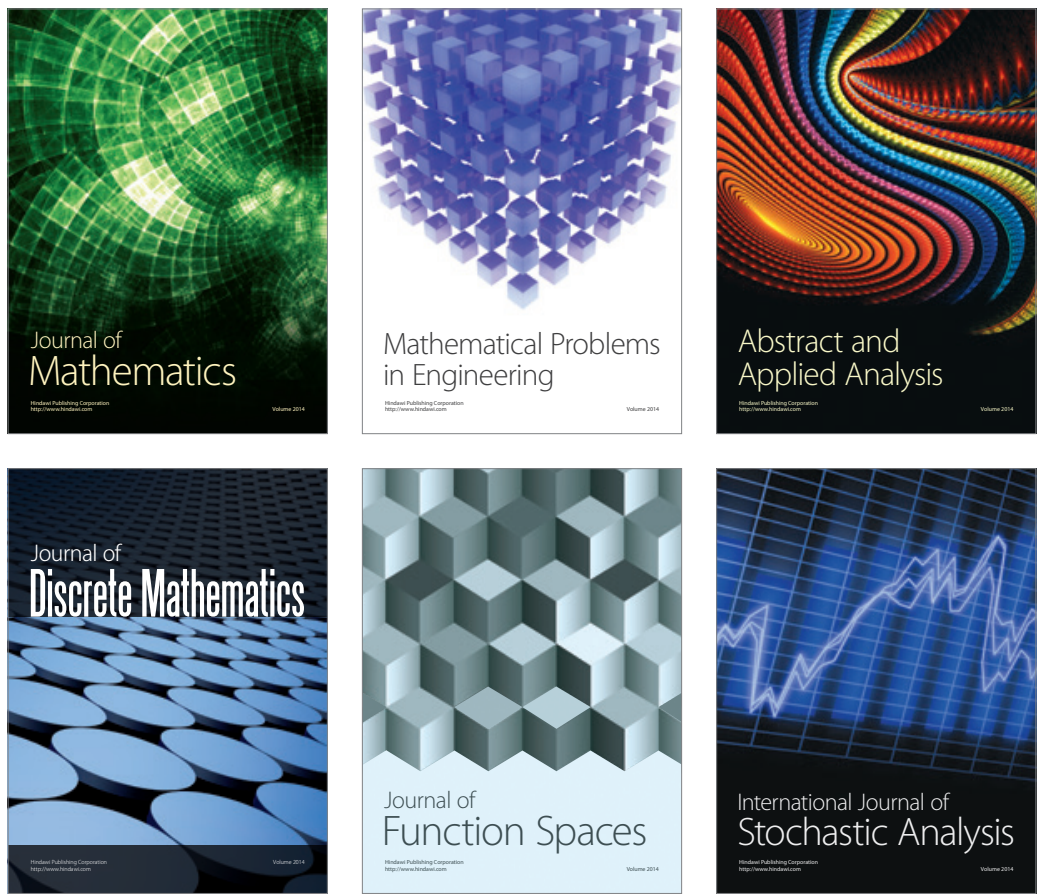

Journal of

Function Spaces

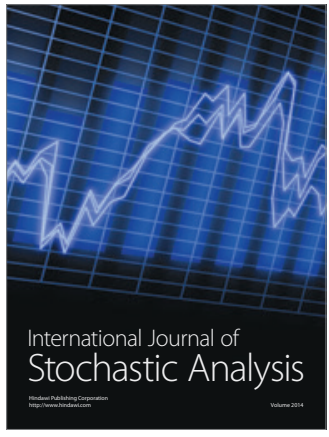

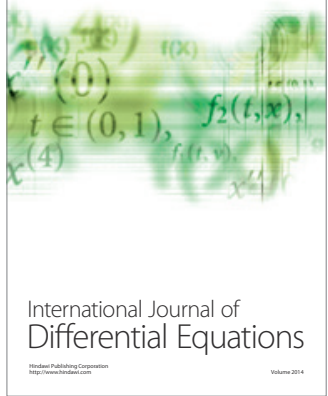
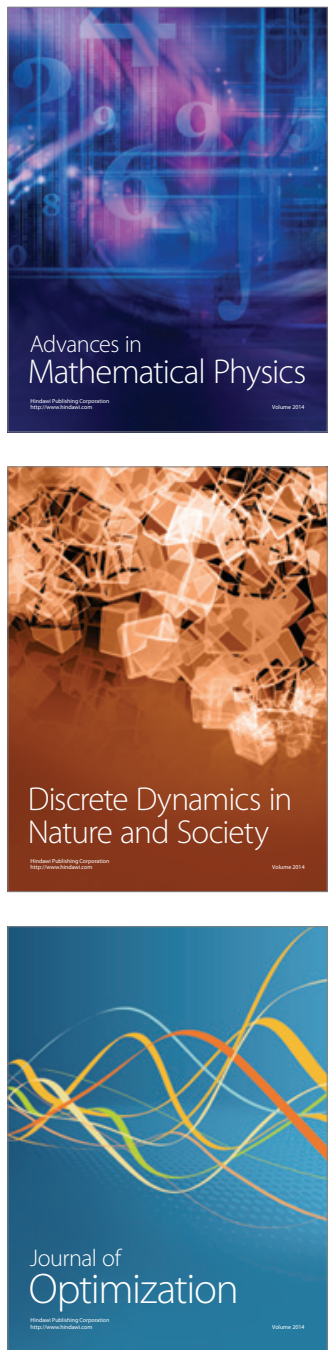